\title{
Analysis of Local Online Review Systems as Digital Word-of-Mouth
}

\author{
Claudia López \\ University of Pittsburgh \\ 135 North Bellefield \\ Pittsburgh, PA 15260, USA \\ cal95@pitt.edu
}

\author{
Rosta Farzan \\ University of Pittsburgh \\ 135 North Bellefield \\ Pittsburgh, PA 15260, USA \\ rfarzan@pitt.edu
}

\begin{abstract}
Using a large dataset of Yelp's online reviews for local businesses, we investigate how Word-of-Mouth research can inform the design of local online review systems and how these systems' data can extend our understanding of digital WOM in a local context. In this paper, we analyze how visual cues currently present in Yelp map to WOM concepts. We also show that these concepts are highly related to the perceived usefulness of the local reviews, which is aligned with prior WOM literature. Additionally, we found that local expertise, measured at the level of the neighborhood, strongly correlates with the perceived usefulness of reviews. Our findings augment the understanding of local online WOM and have design implications for local review systems.
\end{abstract}

\section{Categories and Subject Descriptors}

H.0. [Information Systems]: General

\section{Keywords}

Local online review systems; WOM; interface design.

\section{INTRODUCTION}

Online review systems have created a digital and largescale version of word-of-mouth (WOM) [8]. Websites such as Epinions.com ${ }^{1}$ empower thousands of people to share asynchronously their perceptions about a wide variety of goods and services. More recently, Yelp ${ }^{2}$ and Google Places ${ }^{3}$ have harnessed the power of digital WOM in a local context. They enable residents and visitors to broadcast their opinions about the cities' establishments [5], consequently building a large online source of locally focused information.

While local online review systems broaden access to local information, they also challenge their readers to find relevant

\footnotetext{
${ }^{1}$ http://www.epinions.com/

${ }^{2}$ http://www.yelp.com/

${ }^{3}$ https://plus.google.com/local
}

Copyright is held by the International World Wide Web Conference Committee (IW3C2). IW3C2 reserves the right to provide a hyperlink to the author's site if the Material is used in electronic media. WWW'14 Companion, April 7-11, 2014, Seoul, Korea. ACM ACM 978-1-4503-2744-2/14/04.

http://dx.doi.org/10.1145/2567948.2576933 . information amidst an abundance of data provided by a large pool of strangers. With respect to local knowledge, people would conventionally use traditional media (e.g. newspapers, radio, TV, travel guides) and/or their social contacts (e.g. friends and family) to get the information they need. Even today, people still rely heavily on these sources [15]. These traditional sources provide a more constrained range of information. Editorial and financial restrictions influence what gets published in traditional media. The size and quality of individuals' social networks regulate the amount and types of information obtained through WOM. Additionally, both traditional media and WOM implicitly carry reliability and credibility cues. For example, individuals estimate the relevance and quality of traditional WOM based on the perceived expertise of the information provider [4]. Despite the system designers' attempts to sort out reviews and assist readers in finding the relevant information, many traditional cues such as the information providers' expertise are often lost in the online review systems.

Our research aims to investigate 1) how WOM research can inform the design of local online review systems to better support the readers, and 2) how the data collected by these systems can extend the understanding of local online WOM. In this paper, we report on our findings from a study of a large dataset of Yelp local reviews related to the U.S. state of Arizona. Our findings reveals how design and textual cues present in local online review systems map to prior research in WOM literature. Several cues currently provided by Yelp group into a few major components highlighted by WOM literature as factors influencing the effectiveness of WOM. Our data analysis provides additional evidence that those factors strongly relate to usefulness of local reviews. Additionally, our results show that local expertise is another aspect that is highly correlated with the perceived usefulness of local reviews. The paper also discusses design implications of our results and guidelines for future work.

\section{WORD OF MOUTH}

In marketing, the term WOM has been used to represent the phenomenon in which people voluntarily and informally communicate their perceptions about a given service or product with others [1]. Through WOM, people may acquire additional knowledge about the qualities of goods and can make more informed purchase decisions [3]. The effectiveness of marketing WOM depends on characteristics of both the provider and the receiver of information, characteristics of the relationship between them, the message 
itself, and situational factors such as the risk involved in the purchase decision [18].

The perceived expertise of the information provider is strongly associated with how much WOM influences the receiver. The higher the perceived expertise of the source, the more likely the receiver will pay attention to that information [4]. In terms of characteristics of the relationship, people predominantly seek the opinion of those with whom they have strong ties and those who are similar to them [6]. Information provided by strong ties is perceived as more influential [6]. However, recommendation from weak ties can be equally useful in the context of more technical and less subjective issues [10]. Regarding the content of the WOM, the vividness and strength of the message can also affect the perceived credibility of it. More emotional stories that include details are perceived as being more influential [18].

Early research on WOM highlights the relevance of WOM in choosing local services such as finding a physician [13] and an automotive diagnostic center [12]. WOM is still one of the most popular sources of community news in the US. Fifty-five percent of adults report that they acquire different kinds of local information through WOM on a weekly basis [15]. Although people still rely on traditional WOM to learn about the qualities of local businesses, a large proportion of them report that they also use the Internet to achieve the same goal [17]. Online review systems are one of the web resources for this kind of information.

\section{ONLINE REVIEW SYSTEMS AND LO- CAL EXPERTISE}

Online reviews have been conceptualized as an electronic version of WOM $[8,7]$. In light of this, researchers have studied online review systems to extend their understanding of the WOM phenomenon. They have attempted to assess what motivates users to provide electronic WOM, what makes online WOM effective, and what are the consequences of it on readers' perceptions and purchase decisions. For example, they have found evidence that a higher volume of online reviews increases product sales [9]. Additionally, it has been shown that particular aspects of the reviewers and the review affect its credibility. Furthermore, the strength of the argument and getting confirmation of previous beliefs are associated with higher credibility [7]. In turn, the perceived credibility of reviewers is the most relevant factor predicting online WOM adoption [7].

All the factors affecting WOM's effectiveness, such as strength of ties, expertise and vividness of the WOM messages can be expected to increase the acceptance of local digital WOM. Our first research question focuses on exploring how these factors are represented in the context of local online reviews and how they influence the effectiveness of reviews: $R Q 1$ : Which WOM factors are represented in the context of local online reviews and how they influence the usefulness of reviews?

Research on the specific characteristics of local online reviews is scarce. Antin et al. [2] conducted a survey to understand attitudes toward local review systems and local experts in neighborhoods. They found that local experts and non-experts did not differ meaningfully in their tendencies to contribute to local review systems; however, people who perceive themselves as local experts were more willing to share their local knowledge than those who did not consider them- selves to be local experts. Later, Brown [5] conducted interviews with different local review systems' stakeholders in order to characterize the impact of these systems on their interactions. Based on these interviews, the author concluded that local review sites enable their users to "pre-visit" establishments and neighborhoods before actually visiting them. Additionally, he proposed that online reviews have created a new genre of writing, in which reviewers enjoy providing WOM and influencing others' decisions.

Following the WOM literature, local expertise can impact the effectiveness of local reviews by moderating the characteristics of reviewers, the relationship with the reviewers, and the content of the review messages. In terms of the reviewers' characteristics, local knowledge can be considered to be a form of expertise. In terms of relationship with the reviewers, residents report that they would like to contact local experts to receive local advice [2]. Regarding the content of the message, a local expert's affiliation with their neighborhood may imply a certain level of place attachment. Place attachment refers to the emotional bond of an individual with a particular geographical place or environment [14]. A person's feeling of attachment to a place is correlated with a more positive perception of the place and its inhabitants [16]. This place attachment can also influence the content of the messages. In the context of WOM, place attachment can be translated into more vivid, engaging and positive descriptions of places, their establishments and their people, which in turn can become a more influential and credible WOM message [18]. However, there is no evidence that local experts actually provide more useful reviews than other non-experts residents or visitors. Furthermore, the current design of local review systems does not provide information about the reviewers' local expertise at the level of neighborhood, which can lead to think that it is not considered a relevant cue to be provided to the readers. This led us to our second research question: RQ2: Are reviews provided by local experts judged better in terms of their usefulness?

\section{RESEARCH PLATFORM: YELP}

Yelp is an online review system that offers a platform to share reviews about local businesses. As of 2013, Yelp offers its services in 20 countries and has collected more than 39 million local reviews. Yelp offers a wide range of objective and subjective information about the local businesses in order to help people to learn more about them and facilitate their decision-making. Yelp allows users to share their opinion of local businesses by adding reviews. In each review, a user assigns an overall rating to the business (ranging from 1 to 5 stars) and articulates their opinion in a free-text comment entry. User's reviews are collected on business pages. Each Yelp business' page includes: (1) business' descriptive information, (2) aggregated data of its reviews, and (3) a list of its reviews, including data about the reviewers.

The business information includes working hours, address, categories of business, and other objective business' data. The aggregated measures of the business' reviews include the average rating and the total number of reviews, which are shown at the top of the page. Additional information is available upon extra clicks. For example, there are charts that show the frequency of ratings (from 1 to 5 ) of the business' reviews and the average rating over time.

The list of reviews includes data about the reviews and their reviewers .If a business has more than 40 reviews, the 
list of reviews is broken down in several pages of 40 reviews each. The review data includes the rating (i.e. number of stars), the number of votes the review has received, and the free-text comment. The votes are a measure of the quality of a review. Other users can evaluate a review in terms of its usefulness, or if it is cool or funny. For each review, the overall count of each kind of vote is shown below the review's text. Moreover, the length of the review can also be noted. Although the count of words is not explicitly presented, Yelp shows the complete review, regardless of its length. So readers can see if a review is succinct or lengthy.

Each review is complemented by its reviewer's information. This information includes a picture of the reviewer (if available), and the reviewer's name, city, and state. Moreover, measures of the reviewer's participation in Yelp are featured, including the number of reviews and friends on Yelp, and the Elite status, if they are an Elite user. Elite users are selected every year as the most reputable reviewers in the system [5]. It is also possible to get more information about a user by visiting their user's page. The user page includes the rating distribution of their reviews, the number of types of votes their reviews have received (useful, funny, or cool), the number of times the user has been the first reviewer of a local business, the period of time from their first review, and their top reviewed cities and businesses.

This wide range of cues may assist the readers with evaluating the relevance of a review. However, it is still unclear which of them are indeed relevant for the readers. Furthermore, there is very little information about the reviewers' local expertise that can be particularly important in the context of local online reviews. We will evaluate the role of the available cues and local expertise on the perceived usefulness of local reviews.

\subsection{DATASET}

We utilized the dataset made available through Yelp Data Challenge $^{4}$, which includes reviews for local businesses in Arizona from March 2005 until January 2013. This dataset includes a total of 229,907 reviews provided by 45,981 reviewers for 11,537 local businesses.

Additionally, we used the Google Maps $\mathrm{API}^{5}$ to identify the neighborhood corresponding to the latitude and longitude of the businesses reviewed in the Yelp dataset. The Google API did not have data about some of the businesses' location, especially those in small towns. We were able to gather the neighborhood data for 8,414 businesses.

\section{THE NATURE OF YELP'S REVIEWS}

As expected, the distribution of the local reviews follows a long tail distribution in which most businesses have few reviews and a few businesses have a large number of reviews. On average, an establishment had 20 reviews; however, half of the businesses had six or less reviews (range: 3 - 844). Reviewers' contributions follows the same kind of distribution. The average number of reviews provided by a specific user was five, but half of the users contributed two or less reviews. Only $20.6 \%(9,464)$ of the reviewers added ten or more reviews.

The distribution of the review ratings was positively skewed. Approximately $68 \%$ of the businesses were rated as four or

\footnotetext{
${ }^{4}$ http://www.yelp.com/dataset_challenge

${ }^{5}$ https://developers.google.com/maps/
}

Table 1: Pearson's Correlation matrix of votes

\begin{tabular}{lcrr}
\hline & Useful & Cool & Funny \\
\hline Cool & $0.877^{* * *}$ & 1 & - \\
Funny & $0.726^{* * *}$ & $0.777^{* * *}$ & 1 \\
\hline$* * *: \mathrm{p}<.001$ & &
\end{tabular}

five-star, around $15 \%$ as three-star, and $17 \%$ as two or onestar. It has been suggested that people are more likely to provide WOM when they are either very satisfied or very dissatisfied; therefore, the distribution of ratings is expected to have a U-shape [1]. However, a more recent study has shown that positive WOM is three times more likely to occur than negative WOM [11]. The Yelp data shows an increased tendency to share positive experiences in the case of local reviews, thus providing additional evidence to support the results of the latter study.

Yelp users can explicitly evaluate reviews. Most of the reviews $(64 \%)$ were evaluated at least once. The "useful" votes were the most utilized. Fifty nine percent of the reviews received at least one "useful" vote. Smaller proportions of the reviews were ever voted as cool $(37 \%)$ and funny (30\%). As shown in Table 1, there is a high correlation between different kinds of votes; i.e. a review that received a large number of useful votes, received a large number of funny and cool votes as well. The high correlation holds even when excluding the reviews that have zero votes.

\section{CONCEPTUAL UNDERSTANDING OF CURRENT YELP VISUAL CUES}

As described above, Yelp provides a number of visual cues with information about the business and the reviewer. We are interested in investigating the conceptual meaning behind visual cues represented in the Yelp interface and how these meanings connect to the WOM literature. To identify the main concepts behind Yelp visual cues, we extracted all the features represented by Yelp implicitly or explicitly. We included 19 factors representing aggregated measures of users and businesses (see first column in Table 2). The users' measures comprise the aggregated user data that Yelp showcases, indicating volume and characteristics of their contributions. The factors related to business' measures include central tendency and dispersion measures of the business' ratings and volume of the business' reviews.

We conducted a factor analysis with Varimax rotation to identify a list of uncorrelated factors represented by these 19 variables. Table 2 shows the results of the factor analysis. The variables were grouped into six factors. We highlighted the variables that have higher scores in the table. Based on the loading variables, we have conceptualized the main factors as follows:

- Reviewer's Yelp experience: It conveys the user's level of contribution as a provider of local reviews. It includes overall number of reviews, number of different cities and neighborhoods in which the user has reviewed businesses, number of restaurant's reviews and tenure as Yelp reviewer.

- Reviewer's excellence: It describes the quality of the user's reviews. It involves the number of all the different types of votes received by a user. 
Table 2: Dimension reduction of reviewers and business factors

\begin{tabular}{|c|c|c|c|c|c|c|}
\hline & $\begin{array}{r}\text { Reviewer's } \\
\text { Yelp experience }\end{array}$ & $\begin{array}{l}\text { Reviewer's } \\
\text { Excellence }\end{array}$ & $\begin{array}{r}\text { Reviewer's } \\
\text { Writing style }\end{array}$ & $\begin{array}{r}\text { Reviewer's } \\
\text { Rating pattern }\end{array}$ & $\begin{array}{l}\text { Business' } \\
\text { Approval }\end{array}$ & $\begin{array}{r}\text { Business' } \\
\text { Popularity }\end{array}$ \\
\hline Reviewer's \# of reviews & 0.879 & 0.407 & 0.046 & -0.024 & 0.010 & -0.015 \\
\hline Reviewer's \# of cities & 0.906 & 0.258 & 0.066 & -0.006 & 0.019 & -0.029 \\
\hline Reviewer's \# of restaurants & 0.893 & 0.325 & 0.048 & -0.011 & 0.006 & -0.005 \\
\hline Reviewer's \# of first reviews & 0.611 & 0.387 & -0.003 & -0.043 & 0.036 & 0.017 \\
\hline Reviewer's active days in Yelp & 0.709 & 0.03 & 0.207 & 0.085 & -0.014 & -0.011 \\
\hline Reviewer's \# of useful votes & 0.473 & 0.859 & 0.089 & -0.018 & -0.001 & -0.011 \\
\hline Reviewer's \# of funny votes & 0.32 & 0.903 & 0.088 & 0.006 & 0.006 & -0.005 \\
\hline Reviewer's \# of cool votes & 0.417 & 0.895 & 0.085 & -0.018 & 0.000 & -0.008 \\
\hline Reviewer's average \# of words & 0.109 & 0.124 & 0.929 & 0.035 & -0.006 & 0.027 \\
\hline Reviewer's std. dev. \# of words & 0.154 & 0.052 & 0.921 & 0.129 & -0.001 & -0.01 \\
\hline Reviewer's average rating & -0.074 & 0.006 & -0.105 & -0.837 & -0.129 & -0.008 \\
\hline Reviewer's std. dev. of ratings & -0.054 & -0.018 & 0.043 & 0.871 & 0.036 & -0.041 \\
\hline Business' average rating & -0.024 & -0.002 & 0.002 & -0.072 & -0.922 & -0.010 \\
\hline Business' std. dev. of ratings & -0.06 & 0.021 & -0.017 & 0.085 & 0.657 & -0.048 \\
\hline Business' skewness of ratings & 0.057 & -0.015 & 0.009 & -0.004 & 0.882 & -0.169 \\
\hline Business' kurtosis of ratings & -0.048 & 0.018 & -0.007 & 0.028 & -0.760 & 0.240 \\
\hline Business' ratio of $+/$ - ratings & -0.006 & 0.015 & 0.001 & 0.059 & 0.680 & 0.039 \\
\hline Business' \# of reviews & -0.07 & -0.003 & 0.021 & -0.029 & -0.221 & 0.769 \\
\hline Business' active days in Yelp & 0.042 & -0.010 & -0.005 & -0.006 & -0.054 & 0.846 \\
\hline
\end{tabular}

- Reviewer's writing style: It refers to the pattern of length of a user's reviews. It contains the average and standard deviation of the number of words in all the user's reviews.

- Reviewer's rating pattern: It denotes the pattern of ratings that a user has provided. It includes the average and standard deviation of the ratings that the user has assigned to the reviewed local businesses.

- Business' quality: It characterizes the overall perceived quality of a business. It comprises all the measures related to the ratings that a businesses has received, including average rating, standard deviation, skewness, and kurtosis of rating and ratio of negative over positive reviews.

- Business' popularity: It is related to how popular the business is in terms of number of reviews and the interval of time in which the business has received reviews.

The result of factor analysis suggests that many of the visual cues present in the Yelp system can be grouped together to represent a smaller number of major concepts. A set of these factors, including reviewers' Yelp experience, excellence, and rating patterns represent what is suggested by WOM literature as characteristics of the WOM provider. Writing style characterizes the content aspects of WOM and business approval and popularity relates to situational factors influencing WOM.

\section{LOCAL REVIEWS' USEFULNESS}

Influence, credibility and adoption of WOM are the most studied measures of effectiveness of WOM. In the context of online reviews, these measures can be translated to the extent that a review is perceived as useful by the readers. The usefulness of a given review is a subjective measure dependent upon the context and needs of the readers. As Yelp allows its readers to judge the usefulness of a review, we used the number of times that a review has been voted as useful as our dependent variable.
We used a Poisson regression to identify the relationship between the main components identified in our factor analysis, local expertise and the number of times that a review was voted as useful, controlling for other aspects of each review that are highly visible in the Yelp interface and could also affect the perception of usefulness. The results of the Poisson regression are shown in Table 3. In the model, we included the following variables:

- Variables representing the factor analysis' components: These components measure overall characteristics of reviewers' behavior and general businesses' performance (see Table 2). For each factor, we used the Bartlett factor generated by the factor analysis. As the components represent overall patterns, we had to ensure that our overall measures were not correlated with review-level measures. For users who have provided very few reviews, the overall measures are almost the same as the review-level measures. Therefore, we removed data associated with users with less than five reviews from our dataset. This strategy reduced the size of the dataset to 158,790 reviews.

- A variable measuring the reviewer's local expertise: Local expertise was measured at the neighborhood level for each review. It describes the number of reviews that a user has provided for other establishments in the same neighborhood. Half of the reviews came from users who had no other reviews for businesses in the same neighborhood. However, there are some reviewers who exhibit a trend of local expertise by providing reviews for several businesses in the same neighborhood. Specifically, $25 \%$ of the reviews were written by users who had created between two and four reviews in the same neighborhood, and the remaining $25 \%$ were provided by users who had between five and 96 reviews in the same neighborhood. Given this uneven distribution, we discretized this count variable into an ordinal indicator that has three levels: 'newbies' who are reviewers of only a single establishment in the neighborhood, 'local-fans' who had between two and four reviews in the 
neighborhood, and 'local-experts' who had more than five reviews in the neighborhood.

- Variables describing aspects of a review that are visible in the interface: for each review, we included the rating, the standardized number of words in the review, the number of prior reviews provided by the same user, and the number of prior reviews received by the reviewed establishment.

\subsection{Effect of reviewer and business factors}

The model shows that all the reviewer's and business' factors are significant predictors of a review's usefulness. Reviews that are written by more experienced Yelp reviewers, those that write longer reviews (writing style), and those who generally receive more votes (excellence of reviews) have increased chances to get more "useful" votes. Keeping all remaining variables constant, a unit increase in the reviewer's overall experience is expected to increase their review's votes by $13.4 \%$. One unit increase in users' writing style scale is expected to increase usefulness of a review by $18.8 \%$. A unit of increase in the overall excellence of the user's reviews is expected to increase the ratio of votes by $18.5 \%$. However, reviews that are added by people who have a more positive trend of ratings are expected to receive smaller number of "useful" votes. The count would drop by $3.2 \%$ when there is a unit of increase in this scale.

While the overall popularity of the business is positively related to the usefulness of its reviews, the level of approval is negatively related to it. Reviews of more popular businesses are considered useful more frequently. One unit increase in business popularity is expected to increase the count of "useful" votes by $12.4 \%$. However, the count of votes would drop by $6.7 \%$ when the approval of the reviewed establishment is one unit higher.

\subsection{Effect of local expertise}

The model also shows that local expertise significantly contributes to increases in the number of times that a review is voted as useful.The estimated number of "useful" votes increases $2.1 \%$ when the review was provided by a 'local-fan' that has between two and four reviews in the same neighborhood compared to someone that has added only one review in the business' neighborhood. The magnitude of the increase grows when the review was provided by a 'localexpert'. Compared to a reviewer with only one review in the neighborhood, the count of votes increases $14.3 \%$ when the reviewer has more than five reviews in the neighborhood.

\subsection{Effect of various aspects of a review}

The review's rating is also related to the count of "useful" votes. Reviews with two, three, four and five stars are rated as useful less often than reviews with one star. The rates are expected to drop by $20 \%, 37 \%, 31 \%$, and $19 \%$, respectively. This suggests that readers find reviews with the most negative ratings the most useful. This result is consistent with the traditional belief that negative WOM is more influential [3]; however, it is not aligned with newer studies [11] that have shown that people are less susceptible to negative rather than positive WOM.

Longer reviews are found to be more useful. The usefulness of reviews are expected to increase by $19.9 \%$ when the reviewers write one standard deviation ( 83 words) more than the average length of reviews ( 135 words). This means that one paragraph in excess of the average considerably increases
Table 3: Estimating review's usefulness

\begin{tabular}{ll}
\hline Predictor & $\exp (\beta)$ \\
\hline Intercept & $1.787^{* * *}$ \\
Expertise $=$ local-experts & $1.143^{* * *}$ \\
Expertise $=$ local-fans & $1.021^{*}$ \\
Expertise $=$ newbies & 1 \\
Review rating $=5$ & $0.807^{* * *}$ \\
Review rating $=4$ & $0.694^{* * *}$ \\
Review rating $=3$ & $0.627^{* * *}$ \\
Review rating $=2$ & $0.801^{* * *}$ \\
Review rating $=1$ & 1 \\
Std. length of review & $1.199^{* * *}$ \\
Prior reviewer reviews & $1.001^{* * *}$ \\
Prior business reviews & $0.998^{* * *}$ \\
Reviewer experience & $1.134^{* * *}$ \\
Reviewer writing style & $1.188^{* * *}$ \\
Reviewer rating pattern & $0.968^{* * *}$ \\
Reviewer excellence & $1.185^{* * *}$ \\
Business approval & $0.933^{* * *}$ \\
Business popularity & $1.124^{* * *}$ \\
\hline${ }^{1}:$ reviews $=158,790$, users $=9,685$, businesses $=7,752$ \\
$*^{*}: p<0.05 ; * *: p<0.00 ;$ QIC $=295,792.189$
\end{tabular}

the chances of a review to be voted as useful. Although we did not study the content of the reviews, this result can be connected to prior research that has shown that WOM messages that include more details are more influential [18].

In terms of the remaining of the review variables, (1) late reviews of businesses are less likely to be useful, a review which is added after 10 prior reviews has $2 \%$ less chance of being voted useful; (2) late reviews of reviewers are more likely to be found useful, a review from a reviewer who had added 10 prior reviews is $1 \%$ more likely of being found useful. Although these factors are significant, the magnitude of their effect is very small.

\section{DISCUSSION}

Our research attempts to increase the understanding of how cues provided by Yelp relates to WOM literature. We evaluated the relationship between those cues and the usefulness of reviews. We found that the set of available cues in Yelp can be grouped into six main components of overall reviewer's behavior and business' performance. All of them are significantly related to a review's usefulness.

The relevance of the information provider's characteristics is hardly surprising in the WOM literature. The positive effect of writing style, as well as the length of each particular review, could be related to the kind of detailed information that can be embedded in longer reviews. The tendency of readers to find more credible those WOM messages that convey more vivid stories by including emotions and more details [18] could explain why longer reviews are perceived to be more useful. Content analysis of the reviews is necessary to confirm this hypothesis. The positive relationship of usefulness with more experienced reviewers and users who have other high quality reviews may indicate that better writing skills permit users to provide stronger local reviews, as suggested in [5]. Our findings hint that these skills could also be developed over time. For example, users who spend longer time on the site may be able to develop the necessary skills to write reviews that are perceived as more useful. 
We have also observed that local expertise is significantly associated with perceived usefulness. Despite the influence of local expertise, it is not explicitly presented to readers of Yelp reviews. We speculate that the local expertise effect observed in this study is embedded in the review's text in the form of emotions, shared local background or comparison with other local places that can reveal the reviewer's expertise to the reader and also make the reviews more appealing, as suggested in [18]. Explicit presentation of local expertise can be beneficial to the readers to judge the usefulness of a review. Further research is required to better understand the mechanism that makes reviews by local experts to be more useful. For example, it would be desirable to distinguish the content effect of the message from the effect of the relationship between the reader and the reviewer.

\subsection{Limitations of current study}

As with any study, this research has limitations that must be taken into account when considering its implications. We used the number of times that a review was voted as useful as a reader-reported measure of usefulness of reviews. This measure can be biased because the exposure time of the reviews is not homogeneous. Nevertheless, the exposure time of reviews in Yelp is expected to be long due to the low frequency of new reviews for each establishment, which can help to mitigate the effect of this bias.

Several additional aspects could not be evaluated in this study. The Yelp dataset did not include information about the reviewer's city and Elite status, so we could not include this data in our analysis. We conducted an exploratory analysis (not reported here) to assess the effect of these measures. We collected the city and Elite status of 1,000 users, and found they did not affect our main findings. However, a better estimation could be achieved if this data were available for all the users. Furthermore, the users' social network in Yelp can also be used to assess the effect of weak and strong ties in the credibility of local reviews. Despite these limitations, our findings shed light on aspects of reviewers, businesses and reviews that affect the perception of usefulness of local reviews, which leads us to formulate a number of design guidelines below.

\subsection{Design implications}

We were able to show that local expertise is strongly associated with the number of "useful" votes that a business' review receives. Online local review systems can benefit their readers by featuring local experts. Having a measure of neighborhood expertise in the list of reviews can help readers to learn about the perception of local experts regarding a given establishment. While neighborhood expertise can be obtained explicitly, our results suggest that an implicit measure of local expertise can be a useful alternative measure.

Our analysis also provides guidelines as to what other major features are relevant to the usefulness of local reviews. The reviewers' writing style and rating pattern are strongly associated with the perception of usefulness of their reviews. Local review systems can provide this information by, for example, adding tags to distinguish users whose contribution patterns are associated with more useful reviews. Making this characteristic visible in the list of reviews can help readers to easily profile reviewers, and determine which type of reviewers provides them with more useful reviews.

\section{REFERENCES}

[1] E. W. Anderson. Customer satisfaction and word of mouth. Journal of Service Research, 1(1):5-17, 1998.

[2] J. Antin, M. de Sa, and E. F. Churchill. Local experts and online review sites. In $C S C W$, pp. 55-58, 2012.

[3] J. Arndt. Role of product-related conversations in the diffusion of a new product. Journal of Marketing Research, 4(3):291-291, 1967.

[4] H. S. Bansal and P. A. Voyer. Word-of-mouth processes within a services purchase decision context. Journal of Service Research, 3(2):166-177, 2000.

[5] B. Brown. Beyond recommendations: Local review web sites and their impact. ACM Trans. Comput.-Hum. Interact., 19(4):1-24, 2012.

[6] J. J. Brown and P. H. Reingen. Social ties and word-of-mouth referral behavior. Journal of Consumer Research, pp. 350-362, 1987.

[7] M. Y. Cheung, C. Luo, C. L. Sia, and H. Chen. Credibility of electronic word-of-mouth: Informational and normative determinants of on-line consumer recommendations. Int. Journal of Electronic Commerce, 13(4):9-38, 2009.

[8] C. Dellarocas. The digitization of word of mouth: Promise and challenges of online feedback mechanisms. Management Science, 49(10):1407-1424, 2003.

[9] W. Duan, B. Gu, and A. B. Whinston. Do online reviews matter? An empirical investigation of panel data. Decision Support Systems, 45(4):1007-1016, 2008.

[10] D. Duhan, S. Johnson, J. Wilcox, and G. Harrell. Influences on consumer use of word-of-mouth recommendation sources. Journal of the Academy of Marketing Science, 25(4):283-295, 1997.

[11] R. East, K. Hammond, and M. Wright. The relative incidence of positive and negative word of mouth: A multi-category study. Int. Journal of Research in Marketing, 24(2):175 - 184, 2007.

[12] J. E. Engel, R. D. Blackwell, and R. J. Kegerreis. How information is used to adopt an innovation. Journal of Advertising Research, 9(December):3-8, 1969.

[13] S. P. Feldman and M. C. Spencer. The effect of personal influence in the selection of consumer services, 1965.

[14] G. S. Mesch and O. Manor. Social ties, environmental perception, and local attachment. Environment and behavior, 30(4):504-519, 1998.

[15] L. Rainie, K. Purcell, A. Mitchell, and T. Rosenstiel. Where people get information about restaurants and other local businesses. Tech. report, Pew Research Center, 2011.

[16] C. Rollero and N. De Piccoli. Place attachment, identification and environment perception: An empirical study. Journal of Environmental Psychology, 30(2):198-205, 2010.

[17] T. Rosenstiel, A. Mitchell, K. Purcell, and L. Rainie. How people learn about their local community. Tech. report, Pew Research Center, 2011.

[18] J. C. Sweeney, G. N. Soutar, and T. Mazzarol. Factors influencing word of mouth effectiveness: receiver perspectives. European Journal of Marketing, 42(3/4):344-364, 2008. 